\title{
Recovery of Thyroid Function After 26 years Post Thyroidectomy for Graves' disease with evidence of Active Remnants
}

\author{
Raya Almazrouei, Sara Haboosh, Florian Wernig, Jeannie F Todd \\ Imperial College Healthcare NHS Trust
}

\section{Introduction:}

- Treatment options for Graves' disease include antithyroid drugs, radioiodine ablation and surgery.

- Thyroid surgery for Graves' disease commonly falls into one of three categories: 1) total thyroidectomy, which aims to achieve complete macroscopic removal of thyroid tissue; 2) bilateral subtotal thyroidectomy, in which bilateral thyroid remnants are left; and 3) unilateral total and contralateral subtotal thyroidectomy, or the Dunhill procedure.

- Total thyroidectomy removes target tissue for the thyroid-stimulating hormone receptor antibody. It controls hyperthyroidism at the cost of lifelong thyroxin replacement. Subtotal thyroidectomy leaves a thyroid remnant and may be less likely to lead to complications, however a higher rate of recurrent hyperthyroidism is expected and revision surgery would be challenging

- We present a case of thyroid function recovery more than two decades after subtotal thyroidectomy with active remnants and positive TSH receptor antibodies.

\section{Investigations:}

- Repeat thyroid function sowed TSH of $0.01(0.3-4.2)$ milliunit/L, free $\mathrm{T} 3$ of $4.3(2.5-5.7) \mathrm{pmol} / \mathrm{L}$, free $\mathrm{T} 4 \mathrm{of} 14.5(9-23) \mathrm{pmol} / \mathrm{L}$ and a positive TSH receptor antibody level of $2.9(<0.4) \mathrm{unit} / \mathrm{ml}$.

- She underwent a thyroid ultrasound that showed three hypervascular nodules (thyroid remnants) in the thyroid bed (Fig 1).

- A Tc-99m uptake scan was suggestive of multiple toxic nodules: two large nodules in the left with high increased tracer uptake and another smaller nodule in the right mid pole of the thyroid with low level activity (Fig 2).

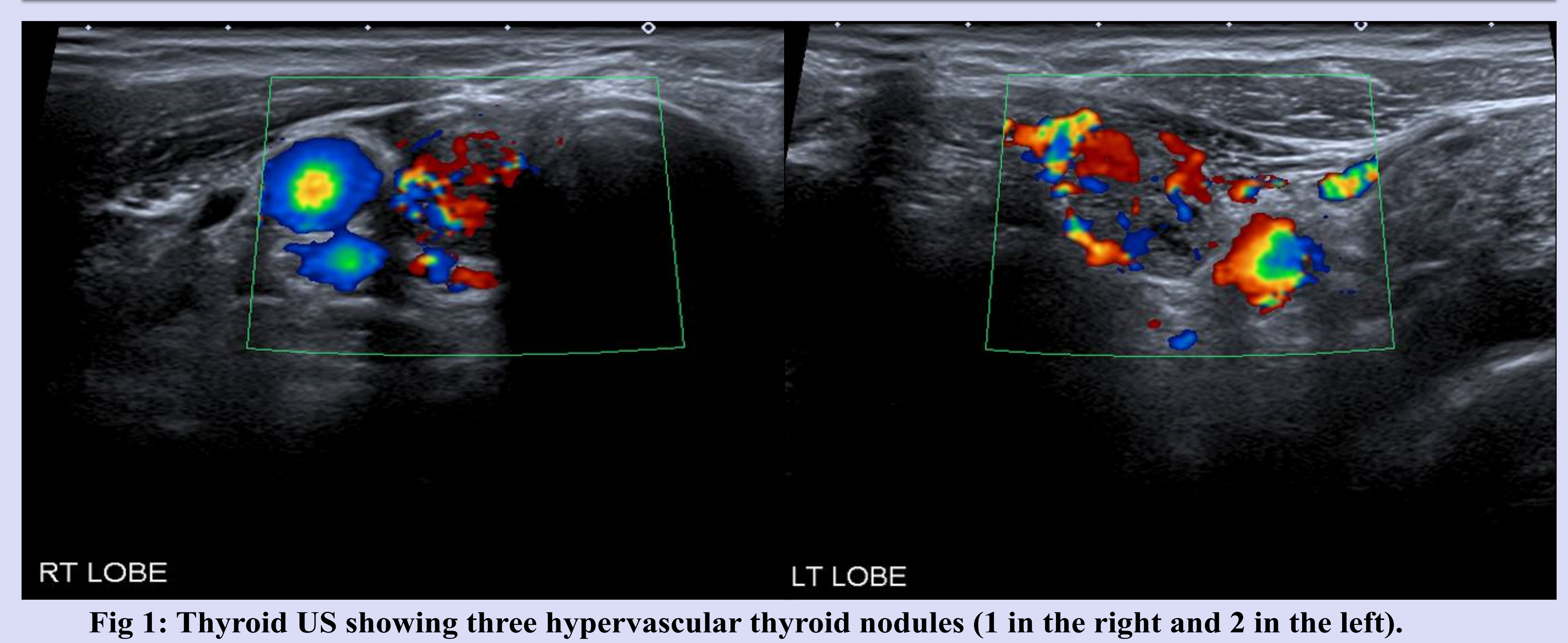

Fig 1: Thyroid US showing three hypervascular thyroid nodules (1 in the right and 2 in the left)

\section{Management:}

- Levothyroxine was withheld In October 2017

- Serial TFT's done subsequently showed evidence of euthyroidism with her latest results in July 2018 (Fig 3) showing TSH of 0.64 (0.3-4.2) milliunit/L, free T4 of 11.7 (9-23) pmol/L and TSH receptor antibody level of $1.5(<0.4)$ unit $/ \mathrm{ml}$.

- She remains under regular surveillance as she is at high risk of Graves disease recurrence.

\section{Case:}

- A 56 year old lady was referred to our endocrine service for further management of levothyroxine replacement.

- She was diagnosed with Graves' disease 26 years ago and underwent subtotal thyroidectomy as definite treatment.

- Post-operatively, she was commenced on $100 \mathrm{mcg}$ of levothyroxine and continued to have regular follow up with her GP.

- It was noted that her levothyroxine dose had to be reduced to $50 \mathrm{mcg}$ daily over a period of 10 years due to persistently suppressed TSH levels with free T4 levels within the normal range.

- In January 2015, her thyroid function showed a picture suggestive of over-replacement with TSH $<0.01$ (0.3-4.2) milliunit/L, free T3 of 6.3 $(2.5-5.7) \mathrm{pmol} / \mathrm{L}$ and free T4 of 24 (9-23) pmol/L. Therefore, her levothyroxine dose was further decreased to $50 \mathrm{mcg}$ on alternate days by her GP.

- In November 2016, she was seen in our endocrine clinic while on the above levothyroxine regimen.

- She didn't report any symptoms related to thyrotoxicosis or overreplacement.

\section{Discussion:}

- In the past subtotal thyroidectomy was commonly performed. Current guidelines recommend that if surgery is chosen as treatment for Graves' disease, total thyroidectomy is the preferred surgery.

- This is due to the fact that total thyroidectomy has a nearly $0 \%$ risk of recurrence, whereas subtotal thyroidectomy may have an $8 \%$ chance of persistence or recurrence of hyperthyroidism at 5 years.

- A recent Cochrane review showed that total thyroidectomy was more effective than subtotal thyroidectomy techniques (both bilateral subtotal thyroidectomy and the Dunhill procedure) at preventing recurrent hyperthyroidism (OR 0.14 (95\% CI 0.04 to 0.46 ) with follow up period range from 6 months to 6 years.

- A recent study from a single centre followed retrospectively 385 patients who underwent bilateral subtotal thyroidectomy and 57 patients who underwent the Hartley-Dunhill operation with median postoperative follow-up time of 72 months (range 12-144 months). This study found that persistent or recurrent hyperthyroidism was observed in $119(28.7 \%)$ patients with euthyroid state achieved in only $19.3 \%$ of patients.

- This case illustrates that clinicians should be aware of the risk of Graves' disease recurrence with previous subtotal thyroidectomy.

\section{References:}

1. Liu et al. Thyroid surgery for Graves' disease and Graves' ophthalmopathy. Cochrane Database of Systematic Reviews 2015 . Issue 11. Art. No: CD010576.

2. Lin et al. The long-term outcomes of thyroid function after subtotal thyroidectomy for Graves' hyperthyroidism. Journal of Surgical Research 2017, 220:112-118. 